\title{
Regenerative Spinal Therapies for Low Back Pain
}

\author{
Beny Charchian - Bianca Tribuzio • \\ Mauro Zappaterra $\cdot$ Mona Zall
}

Published online: 4 February 2014

(C) Springer Science + Business Media New York 2014

\begin{abstract}
With an aging population, chronic low back pain will continue to increase as a source of disability and pain for many patients. This degenerative process can be attributed to a number of diagnoses including spinal stenosis, facet arthropathy, degenerative disc disease (DDD), and herniated nucleus pulposus. These diagnoses have a number of nonregenerative treatment options, including medications, therapy, and traditional interventional procedures. As these treatments are not always successful, novel regenerative treatment options such as platelet-rich plasma (PRP), bone marrow-derived mesenchymal stem cells (MSCs), and growth factors need to be explored. These regenerative therapies are proposed to promote healing, repair, and regeneration of tissue. The use of PRP in spinal fusion has been studied, but there is conflicting evidence affecting spinal fusion rates, and the efficacy is uncertain. PRP and MSCs have been evaluated in the treatment of DDD. Studies show both may have a role in preventing DDD; however, the use of bone marrow-derived stem cells has shown more promising data. This article discusses the available research for regenerative options for the treatment of low back pain. Unfortunately, there is a paucity of literature which examines the use of regenerative medicine as treatment for the wide spectrum of pathologies which cause this common condition. More research is needed to further establish the effective use of PRP, bone marrow-derived stem cells, and growth factors for the treatment of low back pain.
\end{abstract}

\section{B. Charchian}

The Joint and Spine Institute, 9730 Wilshire Blvd. Suite 110, Beverly Hills, CA 90212, USA

B. Tribuzio $(\bowtie) \cdot$ M. Zappaterra $\cdot$ M. Zall

University of California Los Angeles/West Los Angeles

Veterans Affairs, 11301 Wilshire Blvd, Los Angeles, CA, USA

e-mail: biancatribuzio@gmail.com
Keywords Regenerative medicine $\cdot$ Stem cell $\cdot$ Low back pain · Platelet-rich plasma (PRP)

\section{Introduction}

Low back pain has become an increasing cause of concern in the aging population in the United States. Approximately $80 \%$ of individuals will experience back pain at some point in their lives [1]. The most rapid increase is in chronic back pain [2•]. The chronicity has drastically increased health care expenditures, and the economic burden will only continue to grow [2•,3]. A survey by Smith et al. [2•] reports that the outpatient expenditures for chronic back pain has more than doubled from $\$ 15.6$ billion spent in 2000-2001 to $\$ 35.7$ billion in 2006-2007. In most cases, back pain is selflimiting. Studies have shown that patients report improvement of symptoms, pain, and disability within the first 4-6 weeks after an episode of back pain. However, there are a subset of patients who will continue to report pain and disability for up to a year. Others may have a recurrence of their symptoms within the year $[3,4]$. There are currently a variety of treatment options including conservative to interventional treatments. With the growing number of patients, additional and novel options need to be explored. The aim of this paper is to review the evidence of current regenerative treatment options available for low back pain.

\section{Common Causes and Presentation of Low Back Pain}

Low back pain is a vague description of a number of different diagnoses that are primarily related to mechanical factors [1]. These include: muscular strain, spinal stenosis, facet-mediated pain, degenerative disc disease (DDD), and 
radiculopathy $[5,6]$. When assessing patients, it is important to rule out life threatening conditions or pathologies that can be reversible if reached in a timely manner. These conditions include, but are not limited to, infection, cancer, and cauda equina syndrome. Patients with back pain commonly present with symptoms such as burning, aching, shooting, and stabbing pain in the back, and weakness, numbness, and tingling in the legs [6]. Patients with muscular strain typically complain of an aching pain with spasms and have tenderness to palpation. Back pain from spinal stenosis is associated with leg pain that is worse with walking and improved with sitting. Pain associated with herniated discs can cause back and leg pain and paresthesias, and it's worse with forward flexion [6]. Facet-mediated pain is worse with prolonged standing and extension.

\section{Current Nonregenerative Treatment Options}

Current nonregenerative treatment options of symptomatic low back pain include medications, therapy, injections, and invasive techniques such as surgery. First-line pharmacological treatment options include acetaminophen and nonsteroidal anti-inflammatory drugs (NSAIDS) [5]. A variety of NSAIDS may be used, and there is strong evidence that suggests that there is no difference in effectiveness among the medications [7]. Although NSAIDS may be more helpful than acetaminophen [5], there may be serious side effects which include bleeding, perforated ulcers [8], and concern for cardiovascular events $[9,10]$. Muscle relaxants may also be indicated for acute back pain, but have central nervous side effects including fatigue and dizziness. Some muscle relaxants such as carisoprodol have potential for abuse and should be monitored [11]. In addition, mild opioid analgesics may be used for a short duration if patients have debilitating pain that was unsuccessfully treated with first-line therapies [5].

Nonpharmacologic treatments of low back pain include modalities such as heat, ice [12], acupuncture [13], and physical therapy [14], as well as a variety of interventional options [15•]. There continues to be controversy surrounding the efficacy of epidural steroid injections in the treatment of radicular pain [16]. Epidural steroid injections, in particular transforaminal epidural steroid injections, have strong evidence for management of radicular pain from disc herniations [17, 18]. However, these injections may not provide long-term relief of disability and pain [19]. With the use of corticosteroids for these procedures, there is also concern for suppressing the hypothalamicpituitary-adrenal axis [20]. In one study by Ward et al., epidural steroid injections caused impaired fasting glucose and insulin sensitivity for up to 1 week [21]. Moreover, there is also concern for decreased bone mineral density in post-menopausal women receiving these injections. A negative effect was noted on bone mineral density in women who had received over $200 \mathrm{mg}$ of triamcinolone in 1 year [22].

Lately, there has been an increase in reported back pain, and although there are a variety of treatment options, not all of them have been successful in providing long-term relief for patients. As a result, additional treatment options need to be explored. In recent years, research has focused on regenerative treatment options such as bone marrowderived stem cells, platelet-rich plasma (PRP), and growth factors.

\section{Platelet-rich Plasma}

PRP is a biologically active regenerative solution that has gained popularity over the past 20 years in treating a variety of musculoskeletal injuries [23, 24]. PRP is typically prepared from autologous whole blood by centrifugation which separates the platelet-poor plasma and the red blood cells from the middle buffy coat layer containing platelets and leukocytes [24, 25]. The middle layer, or PRP, is separated and consists of a platelet concentration above that of baseline, typically ranging from 2-8 times the normal concentration. The platelets can then be activated using thrombin or calcium chloride, which results in alpha granule release of the endogenous clotting and growth factors found within the platelets [24, 25]. Platelet gel is a slight variation from PRP. It too is derived from autologous whole blood and contains a high concentration of activated platelets in a small volume of plasma, thrombin, and white blood cells. To create the gel, the leukocytes and PRP are combined with thrombin to form a sticky gel [26]. The white blood cell component is rich in neutrophils, monocytes, and myeloperoxidase, which may contribute to bacterial death [26]. Therefore, the PRP solution contains not only platelets and sometimes leukocytes, depending on preparation technique, but also an increased concentration of endogenous growth factors and cytokines known to be important in the healing and regenerative cascade [24].

The activated PRP is a medium containing activated platelets and a myriad of diverse growth factors that are implicated and involved in tissue healing and regeneration. The growth factors contained within PRP have been shown to promote cell proliferation, migration, differentiation, chemotaxis, angiogenesis, regulate inflammation, and promote synthesis and release of extracellular matrix molecules such as collagen that are important in tissue organization and regeneration $[27,28]$. These growth factors include transforming growth factor-beta (TGF- $\beta$ ), basic fibroblast growth gactor (bFGF), platelet-derived growth factor (PDGF), epidermal growth factor (EGF), vascular endothelial growth factor 
(VEGF), hepatocyte growth factor (HGF), insulin-like growth factor-1 and 2 (IGF-1 and IGF-2), and connective tissue growth factor (CTGF) [23, 27, 28]. These growth factors have mitogenic and chemotactic properties that contribute to wound healing, matrix deposition in tendons, and enhancing cellular migration and proliferation [29].

PRP has been investigated as a biologically active regenerative solution for improved bone augmentation [30]. The various growth factors contained in the platelet granules mentioned above play a significant role in bone formation. TGF- $\beta, \mathrm{bFGF}$, and IGF have been shown to stimulate proliferation and differentiation of osteoprogenitor cells. EGF has been shown to stimulate periosteal and endosteal bone formation and VEGF is involved in angiogenesis. In addition to growth factors, activated platelets also release numerous other cytokines and regulatory molecules important in proliferation, differentiation, bone mineralization, and coagulation, which are important for bone regeneration.

For spinal fusions, autologous bone grafting is considered the gold standard, as it provides a scaffold and growth factors for the three properties of bone augmentation including osteoproduction, osteoinduction, and osteoconduction [31]. However, there are some drawbacks to autologous bone grafting, such as increased surgical time, donor site morbidity, infection, vascular and neuronal damage, and limited availability, so that other strategies have been investigated for bone augmentation and improved fusion. One of those strategies is to use PRP to augment bone formation [26].

In a prospective review, Hee et al. [32] showed that the PRP did not increase overall fusion rates, but may provide a faster rate of fusion. In a retrospective study, Lowery et al. [33] showed increased bone formation when PRP was used in conjunction with autografts in lumbar spinal fusions. Hartmann et al. [34] showed that, in spinal stabilization after traumatic fractures of the thoracic or lumbar spine, patients who received bone graft with PRP had increased fusion rates at follow-up compared to the control group that received only bone grafts. Jenis et al. [35] analyzed 22 patients with a 2-year follow up that showed $85 \%$ fusion in the autograft group and an $89 \%$ fusion in the platelet gel and allograft group. The authors concluded that, with similar fusion rates, the platelet gel and allograft can be a safe alternative for autograft. Landi et al. [36], applied the platelet gel to only one side of the operative field when performing a posterolateral fusion, and therefore compared the two sides of the same level to assess for improved fusion. The authors noted increased bone density on the side of the operative field that the platelet gel was used, as well as increased bone apposition at 3 months that normalized at 6 months follow-up, suggesting an increased rate of fusion with the use of platelet gel.
However, not all results have shown PRP to be beneficial. Carreon et al. and Weiner et al. [37, 38] showed decreased rates of fusion with platelet gel when compared to bone graft alone with a 2-year follow-up. Sys et al. [39] noted no improvement or deterioration with PRP compared to autograft alone at 24 months follow-up. Therefore, the role of PRP and platelet gel in spinal fusion is still controversial and uncertain at this time, with more studies needing to be done to determine if specific parameters exist in which it may be shown to have reproducible evidence for its benefit.

Investigators are also looking at the potential role of PRP in DDD [40]. It is thought that intervertebral disc degeneration may initiate in the nucleus pulposus with a shift in the balance between the anabolism and catabolism of cells leading to a decreased deposition of extracellular matrix proteins and increased pro-inflammatory cascade. Intervertebral discs have a rich extracellular matrix, a centrally gelatinous nucleus pulposus surrounded by a more fibrous annulus fibrosis, and cartilaginous endplates. The structural components of the disc come from proteoglycans and collagen. The annulus fibrosis is primarily composed of types I, II, and III collagen, whereas the nucleus pulposus is primarily composed of proteoglycans, but also contains types II and IV collagen [41, 42]. Collagen is important in providing the shape and tensile strength on the disc, while the proteoglycans provide viscoelasticity, stiffness, and resistance to compression through its interaction with water [41]. Degeneration of the intervertebral disc is believed to have multifactorial etiologies, including aging, trauma, excessive mechanical loading, disc morphology, matrix composition, and microenvironment. The subsequent changes include decrease in collagen and proteoglycan synthesis, increase in proteases and cytokines, acidic $\mathrm{pH}$ and increased cell death [42]. Growth factors provide the anabolic components in disc metabolism, while cytokines are responsible for the catabolic effect. Interleukin-1 (IL-1) activates IL6 , nitric oxide, and prostaglandin E2, which are responsible for the breakdown of the proteoglycan matrix [41]. The decrease in the nucleus pulposus seems to be the catalyst for disc degeneration. Therefore, therapeutic strategies have been designed to attempt to increase cell proliferation and secretion of ECM proteins within the nucleus pulposus or to increase anti-inflammatory factors [41]. Delivering therapeutic agents under fluoroscopic guidance to the intervertebral disc may help shift the balance toward anabolic pathways and hinder the progression of DDD. Some of these strategies include injecting stem cells, PRP, and individual growth factors [43].

In vitro studies have shown that PRP and its growth factor milieu can induce human nucleus pulposus cell proliferation and differentiation, and therefore PRP may be useful in preventing DDD [44]. In animal studies, injecting PRP with a gelatin hydrogel which helps immobilize the platelets and growth factors within the nucleus pulposus 
showed a decrease in the progression of DDD in a rabbit model [45]. Similarly, Gullung et al. [46] showed in a rat model of DDD that PRP has a protective effect on the progression of the disease.

\section{Stem Cells}

Adult stem cells are thought to be present in most tissue throughout life, and to provide the basis for tissues maintenance and response to injury. Important adult stem cells include hematopoietic and mesenchymal stem cells (MSCs). MSCs can be derived from bone marrow, adipose tissue, peripheral blood, and embryonic and fetal stem cells [41]. In this paper, we will focus on MSCs derived from bone marrow. Under specific conditions, MSCs can differentiate into myocytes, hepatocytes, neurons, osteoblasts, chondrocytes, adipocytes, ligaments, tendons, fat, and other connective tissues [47, 48]. Moreover, MSCs have been demonstrated to have both anti-inflammatory and immunosuppressive properties.

MSCs have been delivered in a number of ways, including whole blood, marrow concentrates, or ex vivo expanded cell populations. The MSCs are injected or surgically implanted with or without the addition of stimulating factors to the injured tissues [49]. There are several different manufacturing companies that offer kits that separate the bone marrow aspirate into a more concentrated form. The aspirate is usually obtained from the iliac crest. The aspirate volume with anticoagulant is placed in a centrifuge, separating the erythrocytes from nucleated cells and plasma. The plasma and erythrocyte portions are discarded and what is left is the concentrated form [50]. This concentrated form contains growth factors, hematopoietic stem cells, and MSCs.

Growth factors have shown to have regulatory effects on MSCs. Certain growth factors including TGF- $\beta$, IGF-1, IGF-2, and PDGF, as well as the Wnt signaling pathways are important in the promotion of chondrogenesis of the MSCs. Bone morphogenic proteins (BMP) are known to be involved in cartilage formation. They can either act alone or in conjunction with other growth factors to induce chondrogenic differentiation of MSCs [48].

At this time, there are conflicting data with regards to the usage of MSC for the reduction of back pain. Animal studies have shown that injection of MSCs into intervertebral discs have had beneficial outcomes. Sakai et al. [51] showed that when MSCs were injected into rabbits with DDD, the MSCs proliferated and differentiated into a nucleus pulposus-like phenotype. The MSCs were able to produce proteoglycans and type II collagen. Magnetic resonance imaging (MRI) showed improvement in disc height and hydration. Crevensten et al. [52] demonstrated that the implantation of allograft MSCs in rats resulted in increased disc height after a 4-week follow-up. In a prospective case report by Haufe and Mork [53], bone marrow aspirate concentrate was injected into 10 patients who had discogenic pain. The patients were followed for 1 year, and were not allowed to participate in any other therapies at that time. Upon follow-up, none of the patients had any relief, and most ended up having surgery. On the other hand, in a study by Orozco et al. [54], 10 patients who had DDD were injected with autologous expanded bone marrow into the nucleus pulposus. Nine of the 10 patients had significant pain relief and improvement of disability at 3 months, followed by modest additional improvement at 6-12 months. There was no improvement in disc height; however, there was increased water content in the disc as shown by T2-weighted MRI. In addition, Yoshikawa et al. [55] showed that transplantation of a collagen sponge containing marrow MSCs in two females with low back pain provided significant relief of pain at 2 years, and even improved the vacuum phenomenon in the disc as shown by MRI. Moreover, in a retrospective study presented at the American Academy of Pain Medicine, 8 out of 12 patients who were solely injected with bone marrow aspirate concentrate into their disc demonstrated good pain relief at 5-12 months. When they were rechecked at 13-24 months, 5 continued to have significant relief [56].

The use of bone marrow stem cells have been shown to be safe. Hendrich et al. [57०•] looked at the safety of bone marrow concentrate injections in 101 patients with various bone healing disturbances. They found no complications concerning excessive new bone formation, infections, tumor induction, or morbidity at the removal site on the iliac crest.

Based on the above studies, MSCs show great promise in the treatment of DDD. More large-scale studies need to be conducted prior to this becoming the standard of care.

\section{Growth Factors}

Multiple individual growth factors have also been attempted in animal models of DDD [43, 58]. One of the most promising groups of growth factors is the BMP family, which have been found to induce bone and cartilage formation [40]. Clinically, BMP-2 is now used in humans to help improve spinal fusion; however, the results are mixed [59, 60]. BMP-2 has also been shown to regenerate intervertebral discs in animal models [40]. Other growth factors that have been analyzed include IGF-1, growth and differentiation factor-5 (GDF-5), TGF- $\beta$, and osteogenic protein-1 (OP-1) [40]. Based on the evidence from the animal studies, clinical studies assessing the safety and efficacy of intradiscal injections of GDF-5 are underway in the United States [61]. 


\section{Conclusions}

With the increasing number of patients with chronic low back pain and the temporary relief provided by pharmaceutical and interventional procedures, other regenerative treatment options should be considered. PRP, MSCs, and isolated growth factors have shown to be the most promising regenerative therapies. PRP contains platelets, cytokines, growth factors, and other inflammatory modulators that may promote healing and are important in the regenerative cascade [25]. Likewise, bone marrow concentrate contains MSCs and a variety of growth factors and cytokines, which have an immunomodulatory effect on tissue healing and regeneration.

Studies for PRP and growth factors have been performed in spinal fusions and DDD and have speculated that using PRP or growth factors may improve bone fusion and also prevent the degeneration of the disc [45]. However, at this point, the data is lacking to make any definitive conclusions.

The use of the platelet gel and PRP in spinal fusion is still controversial; the literature shows conflicting results. Therefore the efficacy remains uncertain [30,62]. There are still a number of issues regarding PRP that need to be addressed due to the variability of the procedure described [62]. There is no defined platelet concentration that has been determined to be optimal for healing. The various techniques of isolating PRP can lead to vastly diverse platelet concentrations. In addition, there is no clear indication for removing or leaving the leukocytes in the solution. At this point, there is no clear indication for the use of PRP in spinal fusion or DDD, as the literature is difficult to interpret due to differences in study protocols, PRP separation methods, and outcome measures [62].

Similarly, in several studies in patients with DDD, the use of MSCs has resulted in an increase in disc height. However, not all studies have shown that the increased disc height alleviates pain [53-56]. Though the majority of these studies reveal an improvement in pain, the sample sizes were small, and the concentration of the injected MSCs varied from study to study.

In order for PRP, MSCs, or isolated growth factors to become the standard of care for back pain, more large-scale studies are needed. There has been evidence in the use of PRP and MSC for cartilage regeneration for peripheral joints which gives hope that these treatments could be beneficial in the treatment of facet arthropathy, a common cause of axial low back pain. Although regenerative medicine shows a promising future in the treatment of back pain, further research is required to determine the role of regenerative therapies and their use for additional common spine pathologies.
Compliance with Ethics Guidelines

Conflict of Interest B. Charchian declares no conflicts of interest. B. Tribuzio declares no conflicts of interest. M. Zappaterra declares no conflicts of interest. M. Zall declares no conflicts of interest.

Human and Animal Rights and Informed Consent This article does not contain any studies with human or animal subjects performed by any of the authors.

\section{References}

Papers of particular interest, published recently, have been highlighted as:

- Of importance

•- Of major importance

1. Wheeler AH. Diagnosis and management of low back pain and sciatica. Am Fam Physician. 1995;52(5):1333-41.

2. - Smith M, Davis MA, Stano M, Whedon JM. Aging baby boomers and the rising cost of chronic back pain: secular trend analysis of longitudinal medical expenditures panel survey data for years 2000 to 2007. J Manipulative Physiol Ther. 2013;36(1): 2-11. This article is of importance because it shows that, as the population is aging, there will continue to be an increase in the number of patients and the cost of treating low back pain.

3. Heuch I, Foss IS. Acute low back usually resolves quickly but persistent low back pain often persists. J Physiother. 2013;59(2):127.

4. Pengel LH, Herbert RD, Maher CG, Refshauge KM. Acute low back pain: systematic review of its prognosis. BMJ. 2003;327(7410):323.

5. Chou R, Qaseem A, Snow V, et al. Diagnosis and treatment of low back pain: a joint clinical practice guideline from the American College of Physicians and the American Pain Society. Ann Intern Med. 2007;147(7):478-91.

6. Patel AT, Ogle AA. Diagnosis and management of acute low back pain. Am Fam Physician. 2000;61(6):1779-86.

7. Roelofs PD, Deyo RA, Koes BW, Scholten RJ, van Tulder MW. Nonsteroidal anti-inflammatory drugs for low back pain: an updated Cochrane review. Spine (Phila Pa 1976). 2008;33(16):1766-74.

8. Gutthann SP, Garcia Rodriguez LA, Raiford DS. Individual nonsteroidal antiinflammatory drugs and other risk factors for upper gastrointestinal bleeding and perforation. Epidemiology. 1997;8(1):18-24.

9. Hudson M, Richard H, Pilote L. Differences in outcomes of patients with congestive heart failure prescribed celecoxib, rofecoxib, or non-steroidal anti-inflammatory drugs: population based study. BMJ. 2005;330(7504):1370.

10. Solomon DH, Avorn J, Sturmer T, Glynn RJ, Mogun H, Schneeweiss $\mathrm{S}$. Cardiovascular outcomes in new users of coxibs and nonsteroidal antiinflammatory drugs: high-risk subgroups and time course of risk. Arthritis Rheum. 2006;54(5):1378-89.

11. Toth PP, Urtis J. Commonly used muscle relaxant therapies for acute low back pain: a review of carisoprodol, cyclobenzaprine hydrochloride, and metaxalone. Clin Ther. 2004;26(9): 1355-67.

12. French SD, Cameron M, Walker BF, Reggars JW, Esterman AJ. Superficial heat or cold for low back pain. Cochrane Database Syst Rev. 2006;1:CD004750.

13. Vickers A, Zollman C. ABC of complementary medicine. Acupuncture BMJ. 1999;319(7215):973-6. 
14. Clare HA, Adams R, Maher CG. A systematic review of efficacy of McKenzie therapy for spinal pain. Aust J Physiother. 2004;50(4):209-16.

15. - Manchikanti L, Abdi S, Atluri S et al. An update of comprehensive evidence-based guidelines for interventional techniques in chronic spinal pain. Part II: guidance and recommendations. Pain Physician. 2013;16(2):49-283. This article is of importance because it provides evidence-based recommendations for interventional procedures in pain medicine.

16. Staal JB, Nelemans PJ, de Bie RA. Spinal injection therapy for low back pain. JAMA. 2013;309(23):2439-40.

17. Benny B, Azari P. The efficacy of lumbosacral transforaminal epidural steroid injections: a comprehensive literature review. J Back Musculoskelet Rehabil. 2011;24(2):67-76.

18. Schaufele MK, Hatch L, Jones W. Interlaminar versus transforaminal epidural injections for the treatment of symptomatic lumbar intervertebral disc herniations. Pain Physician. 2006;9(4):361-6.

19. Pinto RZ, Maher CG, Ferreira ML, et al. Epidural corticosteroid injections in the management of sciatica: a systematic review and meta-analysis. Ann Intern Med. 2012;157(12):865-77.

20. Kay J, Findling JW, Raff H. Epidural triamcinolone suppresses the pituitary-adrenal axis in human subjects. Anesth Analg. 1994;79(3):501-5.

21. Ward A, Watson J, Wood P, Dunne C, Kerr D. Glucocorticoid epidural for sciatica: metabolic and endocrine sequelae. Rheumatology (Oxford). 2002;41(1):68-71.

22. Kang SS, Hwang BM, Son H, Cheong IY, Lee SJ, Chung TY. Changes in bone mineral density in postmenopausal women treated with epidural steroid injections for lower back pain. Pain Physician. 2012;15(3):229-36.

23. Engebretsen L, Steffen K, Alsousou J, et al. IOC consensus paper on the use of platelet-rich plasma in sports medicine. Br J Sports Med. 2010;44(15):1072-81.

24. Nguyen RT, Borg-Stein J, McInnis K. Applications of plateletrich plasma in musculoskeletal and sports medicine: an evidencebased approach. PM R. 2011;3(3):226-50.

25. Sampson S, Gerhardt M, Mandelbaum B. Platelet rich plasma injection grafts for musculoskeletal injuries: a review. Curr Rev Musculoskelet Med. 2008;1(3-4):165-74.

26. Everts PA, Knape JT, Weibrich G, et al. Platelet-rich plasma and platelet gel: a review. J Extra Corpor Technol. 2006;38(2):174-87.

27. Foster TE, Puskas BL, Mandelbaum BR, Gerhardt MB, Rodeo SA. Platelet-rich plasma: from basic science to clinical applications. Am J Sports Med. 2009;37(11):2259-72.

28. Lee JW, Kwon OH, Kim TK, et al. Platelet-rich plasma: quantitative assessment of growth factor levels and comparative analysis of activated and inactivated groups. Arch Plast Surg. 2013;40(5):530-5.

29. McCarrel T, Fortier L. Temporal growth factor release from platelet-rich plasma, trehalose lyophilized platelets, and bone marrow aspirate and their effect on tendon and ligament gene expression. J Orthop Res. 2009;27(8):1033-42.

30. Miyazaki M, Tsumura H, Wang JC, Alanay A. An update on bone substitutes for spinal fusion. Eur Spine J. 2009;18(6):783-99.

31. Pneumaticos SG, Triantafyllopoulos GK, Chatziioannou S, Basdra EK, Papavassiliou AG. Biomolecular strategies of bone augmentation in spinal surgery. Trends Mol Med. 2011;17(4):215-22.

32. Hee HT, Majd ME, Holt RT, Myers L. Do autologous growth factors enhance transforaminal lumbar interbody fusion? Eur Spine J. 2003;12(4):400-7.

33. Lowery GL, Kulkarni S, Pennisi AE. Use of autologous growth factors in lumbar spinal fusion. Bone. 1999;25(2):47-50.

34. Hartmann EK, Heintel T, Morrison RH, Weckbach A. Influence of platelet-rich plasma on the anterior fusion in spinal injuries: a qualitative and quantitative analysis using computer tomography. Arch Orthop Trauma Surg. 2010;130(7):909-14.
35. Jenis LG, Banco RJ, Kwon B. A prospective study of Autologous Growth Factors (AGF) in lumbar interbody fusion. Spine J. 2006;6(1):14-20.

36. Landi A, Tarantino R, Marotta N, et al. The use of platelet gel in postero-lateral fusion: preliminary results in a series of 14 cases. Eur Spine J. 2011;20(Suppl 1):S61-7.

37. Weiner BK, Walker M. Efficacy of autologous growth factors in lumbar intertransverse fusions. Spine (Phila $\mathrm{Pa}$ 1976). 2003;28(17):1968-70 (Discussion 1971).

38. Carreon LY, Glassman SD, Anekstein Y, Puno RM. Platelet gel (AGF) fails to increase fusion rates in instrumented posterolateral fusions. Spine (Phila Pa 1976). 2005;30(9):E243-6 (Discussion E247).

39. Sys J, Weyler J, Van Der Zijden T, Parizel P, Michielsen J. Platelet-rich plasma in mono-segmental posterior lumbar interbody fusion. Eur Spine J. 2011;20(10):1650-7.

40. Zhang Y, Chee A, Thonar EJ, An HS. Intervertebral disk repair by protein, gene, or cell injection: a framework for rehabilitationfocused biologics in the spine. PM R. 2011;3(6 Suppl 1):S88-94.

41. Longo UG, Papapietro N, Petrillo S, Franceschetti E, Maffulli N, Denaro V. Mesenchymal stem cell for prevention and management of intervertebral disc degeneration. Stem Cells Int. 2012;2012:921053.

42. Huang YC, Leung VY, Lu WW, Luk KD. The effects of microenvironment in mesenchymal stem cell-based regeneration of intervertebral disc. Spine J. 2013;13(3):352-62.

43. Masuda K, An HS. Prevention of disc degeneration with growth factors. Eur Spine J. 2006;15(3):S422-32.

44. Chen WH, Lo WC, Lee JJ, et al. Tissue-engineered intervertebral disc and chondrogenesis using human nucleus pulposus regulated through TGF-beta1 in platelet-rich plasma. J Cell Physiol. 2006;209(3):744-54.

45. Nagae M, Ikeda T, Mikami Y, et al. Intervertebral disc regeneration using platelet-rich plasma and biodegradable gelatin hydrogel microspheres. Tissue Eng. 2007;13(1):147-58.

46. Gullung GB, Woodall JW, Tucci MA, James J, Black DA, McGuire RA. Platelet-rich plasma effects on degenerative disc disease: analysis of histology and imaging in an animal model. Evid Based Spine Care J. 2011;2(4):13-8.

47. Caplan AI. Review: mesenchymal stem cells: cell-based reconstructive therapy in orthopedics. Tissue Eng. 2005;11(7-8):1198-211.

48. Chen FH, Tuan RS. Mesenchymal stem cells in arthritic diseases. Arthritis Res Ther. 2008;10(5):223.

49. Steinert AF, Rackwitz L, Gilbert F, Noth U, Tuan RS. Concise review: the clinical application of mesenchymal stem cells for musculoskeletal regeneration: current status and perspectives. Stem Cells Transl Med. 2012;1(3):237-47.

50. Hendrich C, Franz E, Waertel G, Krebs R, Jager M. Safety of autologous bone marrow aspiration concentrate transplantation: initial experiences in 101 patients. Orthop Rev (Pavia). 2009;1(2):e32.

51. Sakai D, Mochida J, Iwashina T, et al. Differentiation of mesenchymal stem cells transplanted to a rabbit degenerative disc model: potential and limitations for stem cell therapy in disc regeneration. Spine (Phila Pa 1976). 2005;30(21):2379-87.

52. Crevensten $\mathrm{G}$, Walsh AJ, Ananthakrishnan D, et al. Intervertebral disc cell therapy for regeneration: mesenchymal stem cell implantation in rat intervertebral discs. Ann Biomed Eng. 2004;32(3):430-4.

53. Haufe SM, Mork AR. Intradiscal injection of hematopoietic stem cells in an attempt to rejuvenate the intervertebral discs. Stem Cells Dev. 2006;15(1):136-7.

54. Orozco L, Soler R, Morera C, Alberca M, Sanchez A, Garcia-Sancho J. Intervertebral disc repair by autologous mesenchymal bone marrow cells: a pilot study. Transplantation. 2011;92(7):822-8.

55. Yoshikawa T, Ueda Y, Miyazaki K, Koizumi M, Takakura Y. Disc regeneration therapy using marrow mesenchymal cell 
transplantation: a report of two case studies. Spine (Phila Pa 1976). 2010;35(11):E475-80.

56. Meyeer J, Crane D, Oliver K. Lumbar disc biologic autograft injection of bone marrow concentrate for treatment of low back pain: retrospective review of 22 consecutive cases. Paper presented at American Academy of Pain Medicine 2013.

57. • Hendrich C, Franz E, Waertel G, Krebs R, Jager M. Safety of autologous bone marrow aspiration concentrate transplantation: initial experiences in 101 patients. Orthop Rev (Pavia). 2009;1(2):e32. This article is important because it shows bone marrow concentrate has been demonstrated to be safe for patients and did not show any complications concerning excessive new bone formation, infections, tumor induction, or morbidity.

58. Masuda K. Biological repair of the degenerated intervertebral disc by the injection of growth factors. Eur Spine J. 2008;17(Suppl 4):441-51.
59. Michielsen J, Sys J, Rigaux A, Bertrand C. The effect of recombinant human bone morphogenetic protein-2 in single-level posterior lumbar interbody arthrodesis. J Bone Joint Surg Am. 2013;95(10):873-80.

60. Burkus JK, Gornet MF, Schuler TC, Kleeman TJ, Zdeblick TA. Six-year outcomes of anterior lumbar interbody arthrodesis with use of interbody fusion cages and recombinant human bone morphogenetic protein-2. J Bone Joint Surg Am. 2009;91(5):1181-9.

61. (www.clinicaltrials.gov).

62. Sheth U, Simunovic N, Klein G, et al. Efficacy of autologous platelet-rich plasma use for orthopaedic indications: a metaanalysis. J Bone Joint Surg Am. 2012;94(4):298-307. 